\title{
Niobium quarter-wave resonator with the optimized shape for quantum information systems
}

\author{
S.V. Kutsaev ${ }^{1 *}$, K. Taletski1 ${ }^{1,2}$, R. Agustsson ${ }^{1}$, P. Carriere' ${ }^{1}$, A.N. Cleland ${ }^{3,4}$, Z.A. Conway ${ }^{4,5}$, É. Dumur ${ }^{3,4}$, \\ A. Moro' and A.Yu. Smirnov ${ }^{1}$
}

${ }^{*}$ Correspondence:

kutsaev@radiabeam.com

${ }^{1}$ RadiaBeam Technologies LLC,

Santa Monica, USA

Full list of author information is

available at the end of the article

\section{Springer}

\begin{abstract}
Quantum computers (QC), if realized, could disrupt many computationally intense fields of science. The building block element of a QC is a quantum bit (qubit). Qubits enable the use of quantum superposition and multi-state entanglement in QC calculations, allowing a QC to simultaneously perform millions of computations at once. However, quantum states stored in a qubit degrade with decreased quality factors and interactions with the environment. One technical solution to improve qubit lifetimes and network interactions is a circuit comprised of a Josephson junction-based qubit located inside of a high Q-factor superconducting 3D cavity. It is known that niobium resonators can reach $Q_{0}>10^{11}$. However, existing cavity geometries are optimized for particle acceleration rather than hosting qubits. RadiaBeam Technologies, in collaboration with Argonne National Laboratory and The University of Chicago, has developed a niobium superconducting radio frequency quarter-wave resonant cavity (QWR) for quantum computation. A $6 \mathrm{GHz}$ QWR was optimized to include tapering of the inner and outer conductors, a toroidal shape for the resonator shorting plane, and an inner conductor tip to reduce parasitic capacitance. In this paper, we present the results of the resonator design optimization, fabrication, processing, and testing.
\end{abstract}

\section{Introduction}

Nearly all areas of modern life are influenced by the incredible impact of computational capabilities. Quantum computers may make many computationally intense fields of science, such as cosmology, quantum field theory, particle interactions, and nuclear physics, tractable. The building block element of a QC is a quantum bit, which is a two-level quantum system. Qubits enable the use of quantum superposition and multi-state entanglement in QC calculations, allowing a QC to perform millions of quantum mechanical computations at once [1]. Entanglement lets a QC change the state of multiple qubits simultaneously via adjusting the state stored in a single bit, enabling computational power scalability unachievable with traditional computers [2]. These advantages are not just theoret-

(c) The Author(s) 2020. This article is licensed under a Creative Commons Attribution 4.0 International License, which permits use, sharing, adaptation, distribution and reproduction in any medium or format, as long as you give appropriate credit to the original author(s) and the source, provide a link to the Creative Commons licence, and indicate if changes were made. The images or other third party material in this article are included in the article's Creative Commons licence, unless indicated otherwise in a credit line to the material. If material is not included in the article's Creative Commons licence and your intended use is not permitted by statutory regulation or exceeds the permitted use, you will need to obtain permission directly from the copyright holder. To view a copy of this licence, visit http://creativecommons.org/licenses/by/4.0/. 
ical: it was recently reported that quantum supremacy was experimentally demonstrated for the first time using Google's superconducting Sycamore processor [3].

One of the greatest challenges in building QCs is controlling quantum decoherence [4], rapid degradation of the qubit's quantum state due to interactions with the environment and the integrated control channels. Because of decoherence, the relevant quantum behavior is lost, and time-consuming tasks may render some quantum algorithms inoperable [5].

One approach to building a quantum computer is using superconducting RF oscillator circuits with Josephson junctions for anharmonic dynamics [6]. Planar chips allow integrating a large number of qubits in topologies that increase the lifetimes of quantum states by applying error-correction techniques [7]. However, the low intrinsic Q-factors of microstrip resonators limits the coherence times of the qubits [8] while planar geometries require non-trivial solutions for coherence-preserving coupling to the chip $[9,10]$. Another solution to improve qubit lifetimes and allow for network interactions is to couple the Josephson junction through a high Q-factor superconducting 3D cavity [11]. The quantum state excited in the Josephson junction is protected from environmental noise and loss via the encoding qubit states in the high-Q resonant cavity modes [12-15].

The coherence time is closely related to the Q-factor of the resonator and its energy dissipation. Current qubit 3D resonators can achieve $Q \sim 10^{8}$ with coherence times of several milliseconds [16]. On the other hand, niobium resonators used in particle accelerators reach quality factors of $\sim 10^{11}$ [17], potentially enabling storage times approaching seconds [18] if adopted for operation in a QC.

To pursue this opportunity, we developed a 3D superconducting RF (SRF) quarter-wave resonator (QWR) with a shape optimized for high-Q operation in the quantum regime [19] (see Fig. 1). The QWR is an attractive choice, due to the simplicity of its integration with the Josephson junction, which can be placed near the central conductor providing a high coupling strength, and for its feasibility for scaling to multi-qubit systems. The optimization of the cavity includes the introduction of inner and outer conductor tapering,
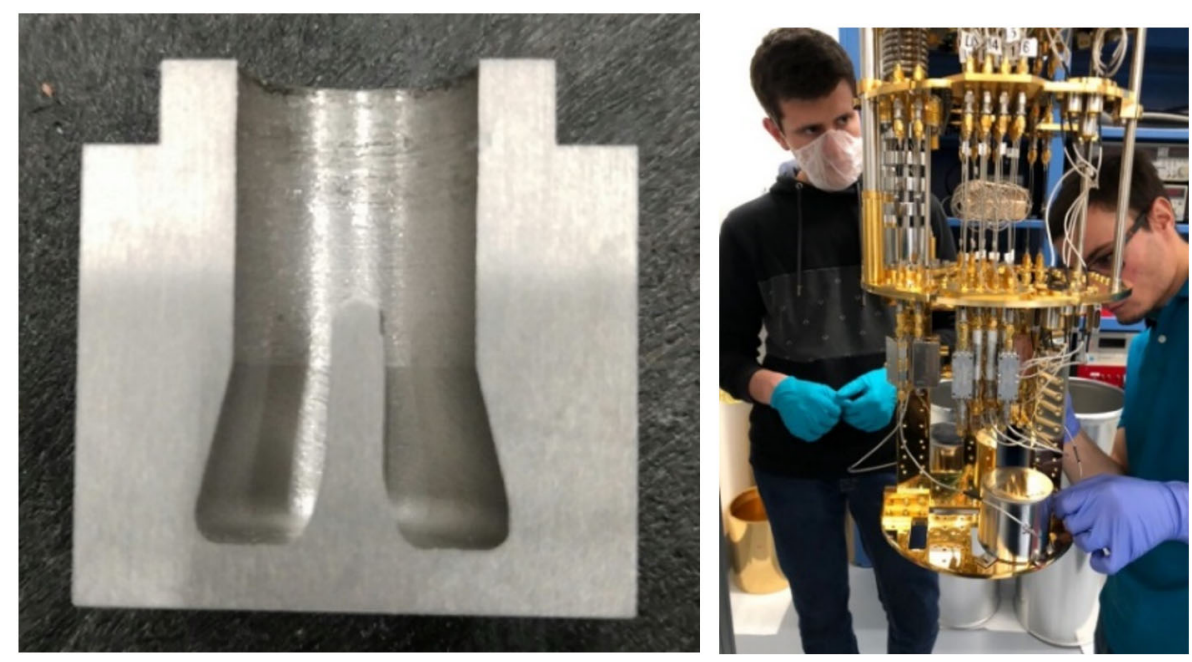

Figure 1 The fabricated 3D niobium QWR cross-section (left), and shielded assembly, attached to the $10 \mathrm{mK}$ stage of the dilution refrigerator (right) 
toroidal shaping of the resonator ends and optimization of the inner conductor tip to reduce parasitic capacitances. These geometrical features provide a better Q-factor and thus a longer lifetime for quantum memory. In addition, we performed a series of numerical simulations and optimizations to decrease energy dissipation in the cavity due to both surface currents and dielectric losses in the niobium oxide layer.

In SRF cavities the unloaded Q-factor can be defined as $Q_{0}=\frac{G}{R_{s}}$. Here, $\mathrm{G}$ is a geometry factor, which ranks the cavity's effectiveness in providing "useful" electric field due to the influence of its shape alone and excludes specific material wall loss. The G-factor can be formally defined as $G=\frac{\omega \mu_{0} \int|\vec{H}|^{2} d V}{\int|\vec{H}|^{2} d A}$, where $\omega$ is the resonant frequency, $\mu_{0}$ is permeability of free space, the top integral is for volumetric RF magnetic field and the bottom is for surface RF magnetic field [20]. Increasing the value of $G$ results in higher $Q_{0}$ and thus indicates a better cavity design. $R_{s}$ is the surface resistance, defined by the material and operating conditions. The surface resistance $R_{S}$ can be expanded into two terms: BCS-resistance and residual resistance, $R_{s}=R_{\mathrm{BCS}}+R_{\text {res }}$. The BCS-resistance is given by the Bardeen-Cooper-Schrieffer theory [21], in which the superconducting Cooper pairs, which have zero resistance for DC current, have finite mass and their momentum alternates sinusoidally due to the AC currents of RF fields, giving rise to energy loss. BCS resistance for niobium depends on frequency and temperature, $R_{\mathrm{BCS}} \sim \frac{A}{T} f^{2} e^{-\frac{\Delta T}{k T}}$ [22], and thus one should keep the frequency and temperature as low as possible to maximize the unloaded Q-factor. The residual resistance arises from several sources, such as material defects, oxides and hydrides that can form on the surface due to hot chemistry and slow cool-down, and other sources related to cavity processing and surface treatment.

\section{Methods/experimental}

\subsection{Geometrical optimization}

As a reference model for comparison of our optimization efforts, we used the straight QWR shape used by Yale [23]. As an initial step for optimization, we adopted the shape of the 72.5 MHz QWR cavity developed at ANL [24]. This geometry was designed for use with high-power accelerating fields with limitations on the maximum surface electric field and surface magnetic fields of $35 \mathrm{MV} / \mathrm{m}$ and $50 \mathrm{mT}$ respectively [25]. These limitations are introduced by electric (E-) field stimulated emission and thermal superconductivity breakdown caused by surface currents induced by the surface magnetic (H-) field. While these surface fields limitations are not relevant for low-power quantum applications, the optimization approach used for these cavities helps to reach better G-factor by reducing peak and integral surface $\mathrm{H}$-fields. We performed further shape optimization by adjusting the geometrical parameters shown in Fig. 2. The simulations were performed in CST Microwave Studio [26].

The machining methods used to produce the cavity put limits on the cavity dimensions. Here we used a milling center to hollow out the cavity resonator from a solid block of niobium. The cutting tool width is constrained by the gap between the inner and outer conductors $w_{b}$ and vibrations experienced during cutting. By analyzing the cutting tool and cavity dimensions (Fig. 3), a simple relation was inferred: $w_{b}=l_{1}+l_{3}+w_{c}$, where $l_{1}$, $l_{3}$ are the outer and inner conductor tapering parameters, and the minimum width of the cutting tool $w_{c}$ is limited by vibrations during machining. This relation generally means that for bigger tapering we will need to increase the gap width. Therefore, we performed simulations to find the dependence of the RF parameters on these dimensions and then optimized them. 


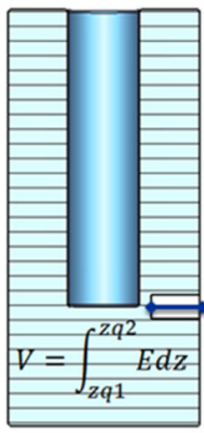

Figure 2 Left: Simple QWR geometry used as a reference. The voltage drop over the transmon (Josephson junction circuit) is highlighted. Right: Initial geometry. Dimensions used for optimization are highlighted

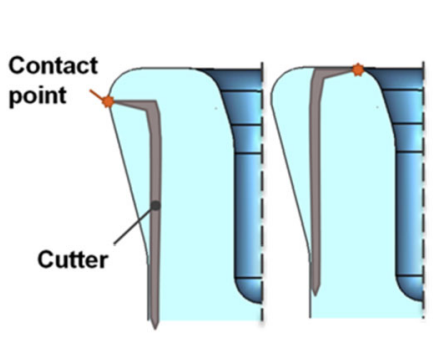

(a)

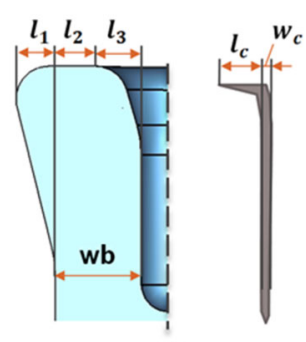

(b)

Figure 3 Machining cutter end positions (a) which define the restriction on gap width $w_{b}$ with the given cavity and cutter dimensions $(\mathbf{b}) I_{1}, I_{3}$, and $I_{c}, w_{c}$ respectively

Figure 4 Distribution of the electric (left) and magnetic (right) fields in the QWR: red—maximum, blue-minimum, linear scale
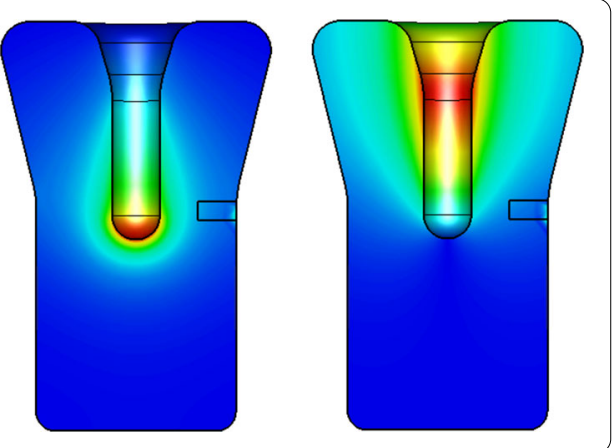

First, we adjusted the geometry of the top part of the resonator. For the QWR geometry, this is the region where the magnetic field energy density is highest (see Fig. 4). By increasing the volume of this part of the resonator, the magnetic energy is distributed over a larger volume and the peak energy density is decreased. Decreasing the magnetic energy density reduces the magnetic surface field and improves the G-factor. We started by simulating geometries with different blending radius of the top part of the cavity. After we explored the outer conductor blending, we optimized the shape of inner and outer conductors by adding tapering. We optimized the taper shape by adjusting the tapering start point for the inner and outer conductors. To maximize the coupling strength with the transmon (Josephson junction), we optimized the inner and outer conductor dimensions, as well as 
the inner conductor radius. Finally, we performed simulations to find the optimal width of the gap between the inner conductor tip and outer conductor wall. The simulated G-factor dependences on the geometric parameters are presented in Fig. 5.

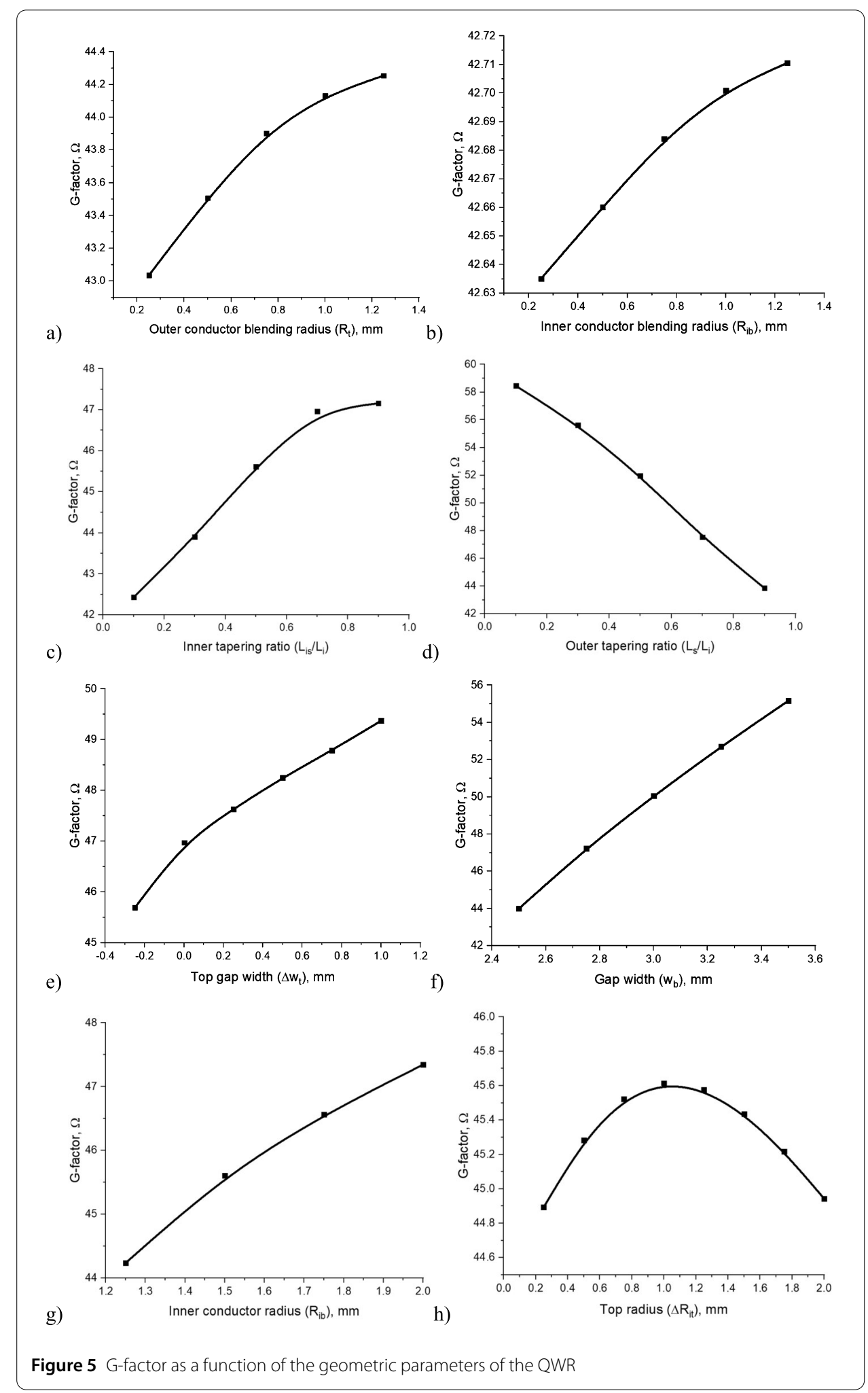


Turning to geometrical optimization to reduce $R_{s}$, the primary source of residual resistance is the thin dielectric layer that is usually present on the surface of superconducting niobium. As shown in recent work at Fermi National Accelerator Laboratory [27, 28], removing this layer can improve the Q-factor by an order of magnitude. Losses in the dielectric layers can be calculated by integrating the E-field over the thin surface dielectric volume:

$$
P_{\text {diel }}=\frac{1}{2} \int_{V_{d}} \overline{J E}^{*} d v=\frac{\omega \varepsilon^{\prime \prime}}{2} \int_{V_{d}}|\bar{E}|^{2} d v
$$

where $\omega$ is the working frequency and $\varepsilon^{\prime \prime}$ is the imaginary part of complex dielectric permittivity. By creating a more uniform E-field distribution, we can potentially lower the surface layer integral and thus decrease the dielectric loss. The E-field is concentrated at the end of the quarter-wavelength loading element and concentrated by the sharp edges of the straight geometry of the central pin as shown in Fig. 4. We blended this feature to achieve a more uniform E-field distribution. Full blending of the inner conductor tip helped to reduce the E-field integral by $22 \%$, thus significantly reducing this source of dielectric loss.

We further investigated the dielectric loss using simulations of a thin $\mathrm{Nb}$ oxide layer on the cavity surface. The prevailing surface oxide in niobium $\mathrm{RF}$ cavities is $\mathrm{Nb}_{2} \mathrm{O}_{5}$ [29]. $\mathrm{RF}$ measurements in thin $\mathrm{Nb}_{2} \mathrm{O}_{5}$ films [30] have found a dielectric constant of about 50 with a loss tangent of 0.01 for temperatures below $\sim 100 \mathrm{~K}$. The results presented in [31] show that the loss tangent remains constant for frequencies larger than $1 \mathrm{MHz}$. Thus we used these parameters in simulations to estimate the upper limit of the Q-factor.

The typical thickness of the oxide dielectric film in niobium RF cavities is around $50 \AA$ [32]. This is much smaller than the cavity dimensions, which are on the order of millimeters. Thus such thin films cannot be simulated directly. Instead, we simulated losses in a thicker $1 \mu \mathrm{m}$ layer, and divided the simulated by a factor of 200 to approximate the expected actual film thicknesses. By doing this, we assumed that the E-field density does not change in this thin oxide film. Inferred dielectric losses thus should depend linearly on the surface thickness $h$ :

$$
\begin{aligned}
& P_{\text {loss }} \propto \iiint \vec{E} \vec{D} d V_{d}=\iint h \vec{E} \vec{D} \cdot d S_{s}, \\
& P_{\text {loss }} \propto h .
\end{aligned}
$$

In these simulations, as we are interested in the Q-factor as limited by dielectric losses, we assumed infinite electric conductivity for the cavity walls. The results of these simulations showed that a cavity with a $50 \AA$ thick $\mathrm{Nb}_{2} \mathrm{O}_{5}$ layer in the optimized geometry almost two times better than the simulated dielectric Q-factor the non-optimized QWR geometry.

We then studied how to reduce the RF losses in the joints between the different components of the cavity assembly. Our fabrication method introduces limits on the dimensions of the cavities. One of the most substantial restrictions is the cavity length. From a manufacturing point of view, it is desirable to make the cavity as short as possible. The shorter cavity allows using a shorter cutter (Fig. 3), reducing vibrations and improving the quality of the machined surface. 
Figure 6 RF volume of the optimized cavity with an opening for vacuum pumping. Niobium cavity (green) attached to an aluminum extension part (blue)

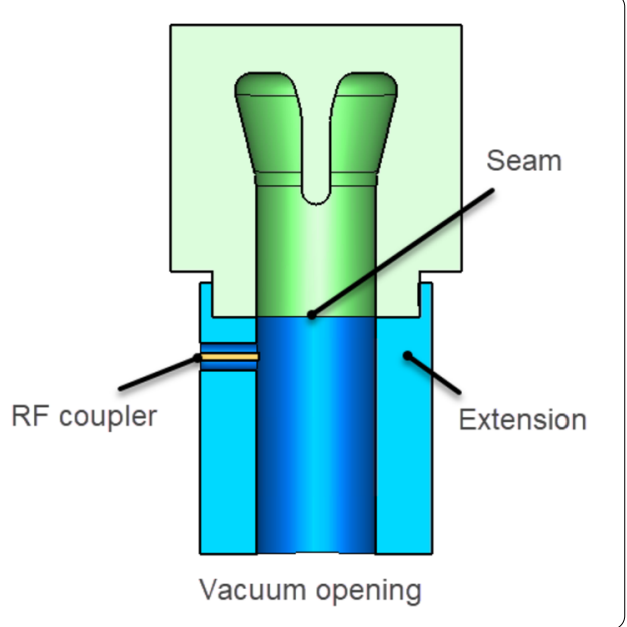

Figure 7 Dependence of the assembly's (left) Q-factor on the cylindrical cavity length $L_{g}$ for different values of niobium conductivity $\sigma$. Copper conductivity is $\sigma_{c} 4=5.51 \cdot 10^{10} \mathrm{~S} / \mathrm{m}$ [33]

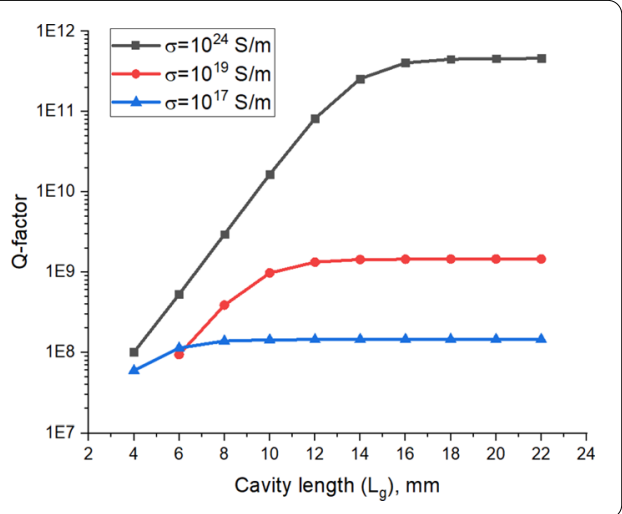

We thus introduced the concept of machining a short cavity elongated by attaching an additional part (Fig. 6) made of a different material. An obvious material choice is aluminum, as this becomes superconducting at $1.2 \mathrm{~K}$, well above our $10 \mathrm{mK}$ operating temperature.

Our new design added a number of parameters for optimization. We performed simulations to define the minimum distance from the niobium inner conductor to the niobium to aluminum connection $\left(L_{\mathrm{g}}\right)$ shown in Fig. 7. The next step was to simulate the joint losses. The surface magnetic field in the region of the QWR joints drives currents across the seam. Lower seam conductivity introduces additional losses; therefore we tried to minimize the H-field on the seam.

One design solution to reduce the joint loss is an RF choke (Fig. 8(e)). The addition of the choke, however, introduces difficulties with the cavity coupling, where the deep choke groove pushes the RF coupling pin (see Fig. 6) too far from the cavity, making the coupling between the cavity and RF transmission line unacceptably weak. On the other hand, a big concern was that coupling through the choke may introduce parasitic modes. This is inacceptable for qubit cavities, which require a clean spectrum for single mode operation. Moreover, eigenmode simulations showed a lower-order mode with E-field concentrated inside the choke. The appearance of a lower-order mode was a significant drawback, and the choke could not be used. 
(a)

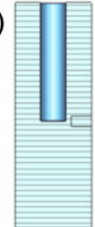

(b)

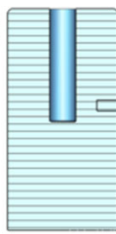

(c)

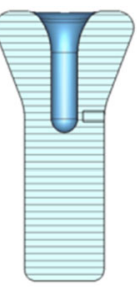

(d)

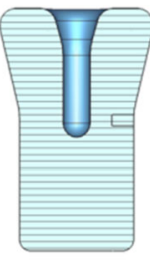

(e)

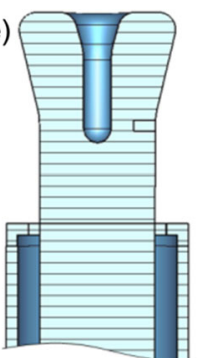

Figure 8 Designs evolution: reference model (a), optimized straight geometry (b), optimal design not feasible with machining (c), final design optimized for machining fabrication (d) and design with RF choke (e)

Table 1 RF parameters comparison of designs elaborated during the optimizations ( $c-d)$ to the reference designs $(a, b)$

\begin{tabular}{llllll}
\hline Design & $\mathrm{a}$ & $\mathrm{b}$ & $\mathrm{c}$ & $\mathrm{d}$ & $\mathrm{e}$ \\
\hline R/Q, $\Omega$ & 46.8 & 54 & 77 & 55 & 55 \\
G-factor, $\Omega$ & 44 & 57 & 62 & 71 & 71 \\
H-field on seam, a.u. & 1 & 3.5 & 0.1 & 2.9 & $10^{-3}$ \\
\hline
\end{tabular}

Figure 9 Full assembly with 2 layers of magnetic shielding, IR cup shield, SMA penetrations and cavity

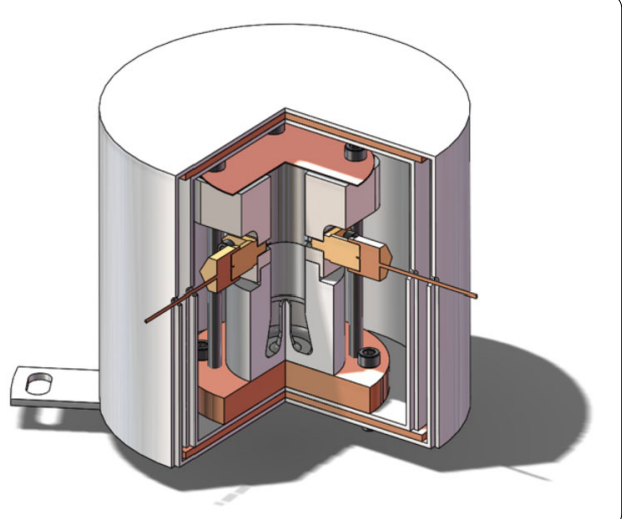

The evolution of the design during the optimization process is illustrated in Fig. 8 and compared quantitatively in Table 1; we managed to increase the G-factor by $65 \%$. Further optimization seems to be possible; however, those designs are not feasible with machining fabrication.

\subsection{Engineering and fabrication}

In order to test this approach, we engineered and built a proof of principle prototype. The engineering design of the prototype includes system integration, thermal management, magnetic shielding, vacuum considerations, and signal integration. The complete system is shown in Fig. 9. Starting from the inside out, the original full-length cavity was truncated to have a separate lower field region made from aluminum that would also provide connection points for the RF signals through non-magnetic SMA connectors. Then considerations were made for sufficient thermal contact and choice of fastener. This was accomplished both through analytical calculation and computational stress analysis in ANSYS Multiphysics [34], as seen in Fig. 10, shown with magnified deformation scales. 


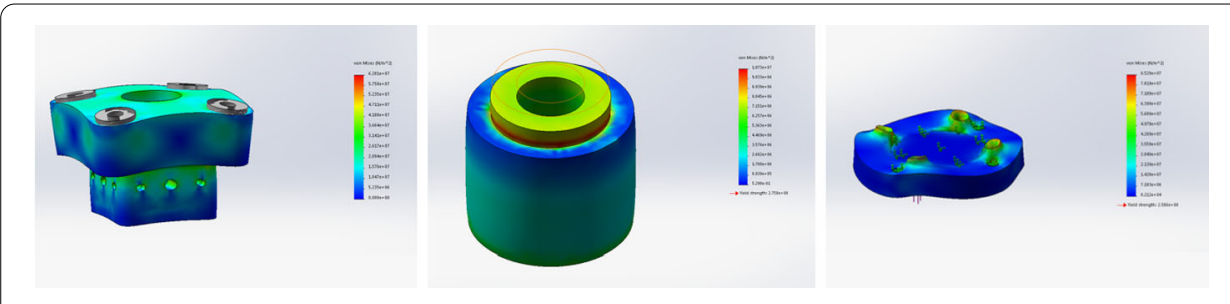

Figure 10 Stress analysis results of the top SMA connection hat, the main Niobium cavity and bottom copper support plate

Next, a simple magnetic shield was designed in Cryoperm 10 [35], a unique cryogenically friendly steel alloy that retains functional permeability at cryogenic temperatures more effectively than conventional MuMetal [36]. This magnetic shield required two separate shielding cans, each manufactured from $1 \mathrm{~mm}$ thick Cryoperm 10, that are separable for assembly of the device under test while permitting small penetrations for SMA cables. Finally, the full system was re-analyzed to ensure that the additional material did not affect the original assembly thermal calculations and provisions were made to ensure the full system could be mounted onto the available test plate within a dilution refrigerator operated at $10 \mathrm{mK}$. At this stage, spring washers were incorporated into the design, and a torque value and target compression were calculated and provided to technicians for assembly.

Regarding the fabrication of the cavity, we encountered challenges during niobium machining that were amplified by over-annealing of the niobium by the vendor. This resulted in a 'gummier' metal consistency, which leads to surface burnishing rather than clean shearing of material and may have limited the $\mathrm{Q}$ of the prototype.

Optical measurements were made on the first fabricated prototype cavity. All dimensions of the cavity were within the design tolerances except for the inner conductor length and inner conductor tilt. The former was decreased by $270 \mu \mathrm{m}$ which, according to simulations, could lead to a frequency increase of $140 \mathrm{MHz}$. Inner conductor tilt was 1.6 degrees and, according to simulations, could not disturb RF parameters of the cavity. Furthermore, this tilt could be due to pressure applied to the sample during the cut for inspection.

We used optical measurements to estimate surface roughness on the outer conductor tapering which was one of the most challenging surfaces from a fabrication point of view. The measured profile is presented in Fig. 11. The surface roughness estimate based on this measurement is $20 \mu \mathrm{m}$ which, however, could be up to $30 \mu \mathrm{m}$ due to the high error of optical measurements of surface profiles.

Two solid niobium $6.2 \mathrm{GHz}$ quarter wave resonators were cleaned, etched and high pressure rinsed at Argonne National Laboratory (see Fig. 12). Figure 13 shows the cavities before and after etching. Both cavities were processed according to the following procedure: (1) a 1 hour ultrasonic cleaning in a $40^{\circ} \mathrm{C} 4 \%$ alconox solution [37]; (2) a ultra-high purity water rinse; (3) dried with filtered and de-ionized nitrogen boil-off gas; (4) a 150 minute buffered chemical polished (BCP) in 1:1:2 ( $\left.\mathrm{HF}: \mathrm{HNO}_{3}: \mathrm{H}_{3} \mathrm{PO}_{4}\right)$; (5) a ultra-high purity water rinse; (6) another 1 hour ultrasonic cleaning at $40^{\circ} \mathrm{C}$ in a $4 \%$ alconox solution; (7) a ultra-high purity water rinse; (8) a high-pressure ultra-high purity water rinse; and finally (9) air dried in class 10 clean room and bagged in a class 100 clean room for transfer to the cryogenic laboratory. 

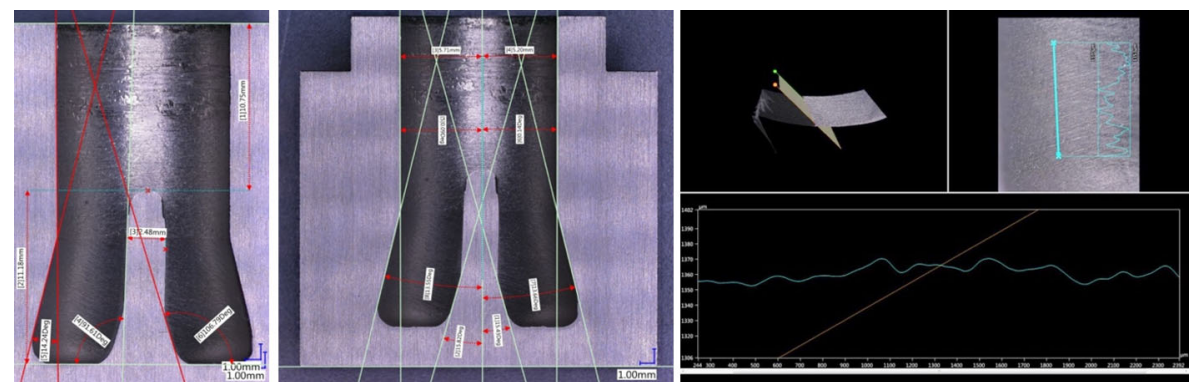

Figure 11 Optical measurements of dimensions (left and middle) and surface profile (right) of the first niobium prototype cavity
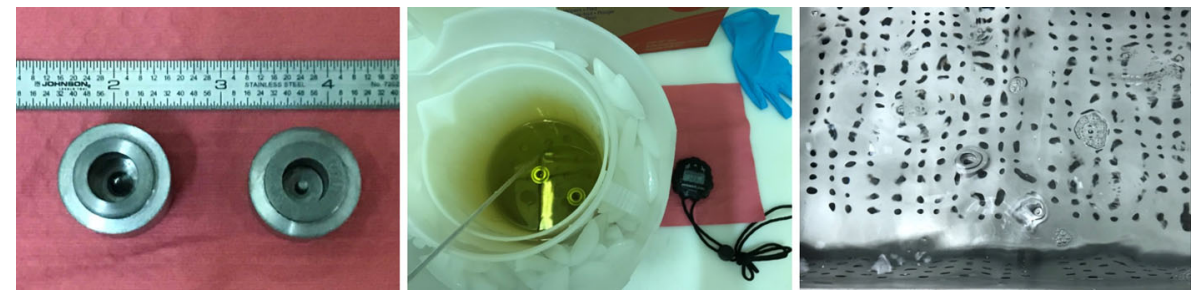

Figure 12 Left: Machined resonators before an etching; Center: Resonators being etched. Etch time $=83$ minutes; Right: resonators in the ultrasonic cleaner after etching
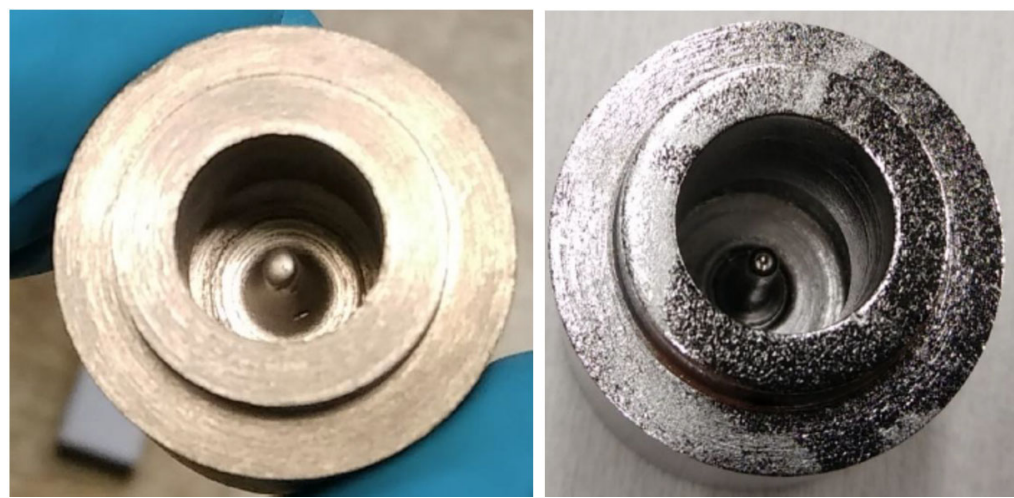

Figure 13 Pictures of the niobium resonator prototype before (left) and after etching (center)

\subsection{Resonator tests}

We performed a series of room temperature (RT) measurements for all fabricated cavities to keep track of their performance. First, we measured the RT quarter-wave resonant frequency and Q-factor (Fig. 14, left). We used a single coupling pin (Fig. 14, middle) to perform reflection-type measurements. The pin length was chosen to be $4.1 \mathrm{~mm}$ above the aluminum part edge to provide sufficient coupling. During the experiment, the cavity was tightly clamped to the aluminum part (Fig. 14, right), until a plateau in the measured Q-factor was observed.

We started from measuring the magnitude of $S_{11}$ (Fig. 15, left), which gave us information about the resonant frequency, $f_{0}=6160 \mathrm{MHz}$. We used the Smith chart representa- 

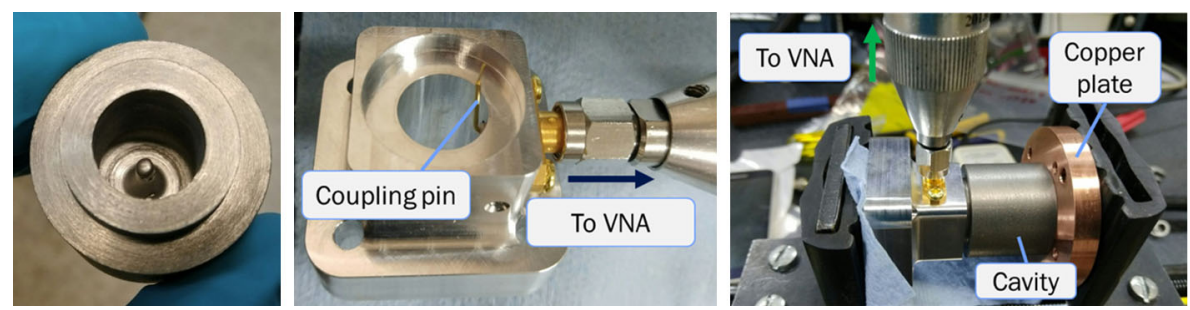

Figure 14 Photos of the first niobium cavity (left) and experiment setup (middle, right)
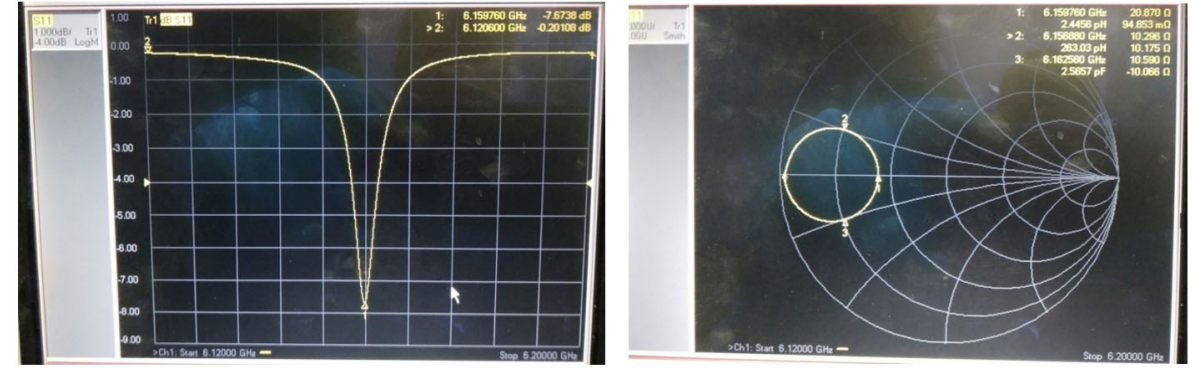

Figure 15 Frequency domain (left) and Smith chart (right) representations of the $S_{11}$ parameter measurements

Table 2 Measured RF parameters of the different prototype cavities at room temperature

\begin{tabular}{lllll}
\hline Material & $\mathrm{Cu}$ & $\mathrm{Nb}$ & $\mathrm{Nb}$ & $\mathrm{Nb}$ \\
\hline Shape & Optimized & Simple & Optimized* & Optimized \\
Resonant frequency $f_{0,}, \mathrm{MHz}$ & 6001 & 6015 & 6160 & 5851 \\
Internal Q-factor & 2631 & 885 & 550 & 1084 \\
\hline
\end{tabular}

*First prototype.

$\dagger$ Second prototype.

tion (Fig. 15, right) to determine the frequencies $f_{1}$ and $f_{2}$ where the real and imaginary parts of the load impedance were equal. These values then were used to calculate the internal Q-factor of the cavity

$$
Q_{0}=\frac{f_{0}}{f_{2}-f_{1}} .
$$

Measurements of the non-optimized and optimized niobium and copper cavities are summarized in Table 2 . The non-optimized niobium cavity appeared to be detuned by $+15 \mathrm{MHz}$, while the optimized cavity with $400 \mu \mathrm{m}$ shorter inner conductor was detuned by $-150 \mathrm{MHz}$, which was $100 \mathrm{MHz}$ less than expected. In order to implement the S11curve measurement method, we added a circulator to the excitation port to measure the reflected power (we note that the unshielded magnetic fields from the circulators may affected the measured Q-factor of the resonators).

In order to accurately calculate the internal Q-factor $Q_{0}$ of the cavity, the external Qfactors $Q_{\mathrm{EXT}}$ of the probes should differ (relative to $Q_{0}$ ) by no more than by an order of magnitude, and ideally by no more that a factor of two [38]. However, since the $Q_{0}$ of the cavity was initially unknown, we had to perform a series of measurements of loaded 

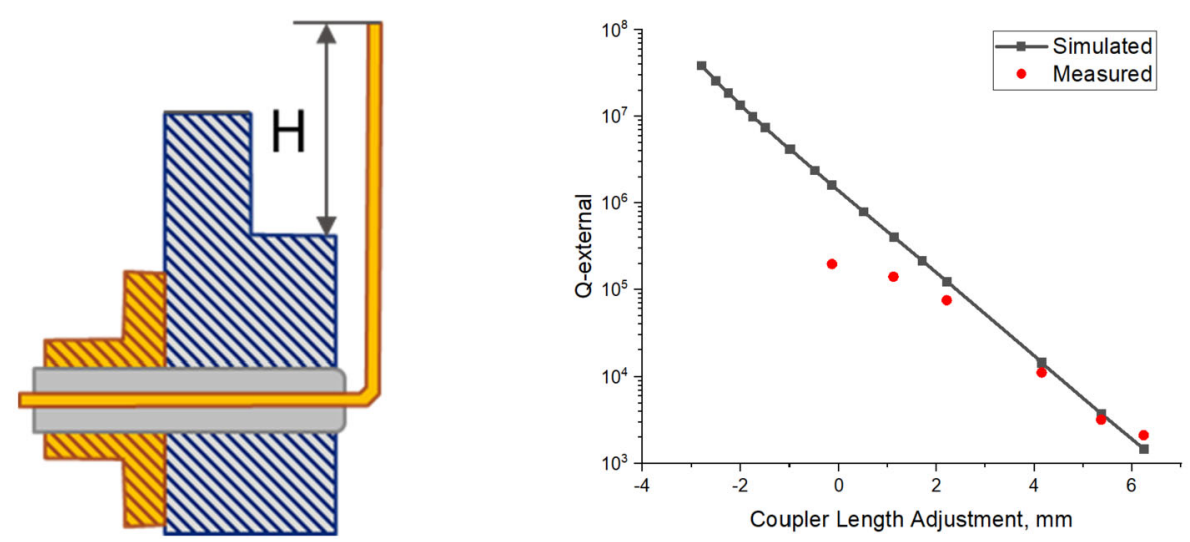

Figure 16 Left: cross section of aluminum part with coupler dimension highlighted; Right: measured and simulated dependences of external Q-factor on coupler length

Q-factor $Q_{L}$ with different external Q-factors $Q_{\mathrm{EXT}}$ of the probes, i.e. the coupling pin of various lengths (Fig. 16, left). For these measurements, a copper cavity with already measured internal Q-factor (at room temperature) was used, so we just calculated external Q-factor for each coupler length by inferring the coupling coefficient from $S_{11}$ measurements. However, since the room temperature $Q_{0} \sim 10^{3}$, these measurements could only be done for $Q_{\mathrm{EXT}} \sim 10^{5}$. For shorter couplers, we used electromagnetic simulations (Fig. 16, right).

In order to measure the Q-factor of the resonator, we adopted the ringdown technique that is widely used for measurements of superconducting accelerating cavities [39]. In this method, the resonant cavity is driven by a short rf pulse into one port. Then the exponentially decaying cavity signal coming out of the second port is captured by an oscilloscope where we measure the decay time. One method of exciting the cavity is with positive feedback or a self-excited loop (SEL) [40], which works without any external rf reference matched to the cavity resonance.

The schematic of the SEL circuit built for our tests is shown in Fig. 17. When the rf switch is in the on state, the rf signal is amplified by a low-noise amplifier (LNA) from a noise that further on gets filtered by a combination of the test cavity itself and an additional band-pass filter. As this signal fills up the cavity the switch turns off and the stored energy decay time is measured by the scope. This method has the advantage of being independent of resonant frequency due to the microphonics, which is essential for measuring high-Q cavities with extremely narrow bandwidths.

The initial tests of the ringdown circuit were carried out on a $6 \mathrm{GHz}$ copper test resonator (see Fig. 18). Typical waveforms are shown in Fig. 19. The decay time $\tau$ was measured, and then the loaded Q is calculated using $Q_{L}=\tau \pi f$, where $f$ is the cavity resonant frequency.

Measurements of the loaded Q using S-parameter measurements in the frequency domain using a vector network analyzer (VNA) showed $Q_{L}=1500$, which was in good agreement with the results of the ringdown measurements that showed $Q_{L}=1550$ ( $\tau \sim 82 \mathrm{~ns}$ ). The SEL-based ringdown measurement circuit proved to work well and matched the results from the frequency domain measurements at room temperature. 


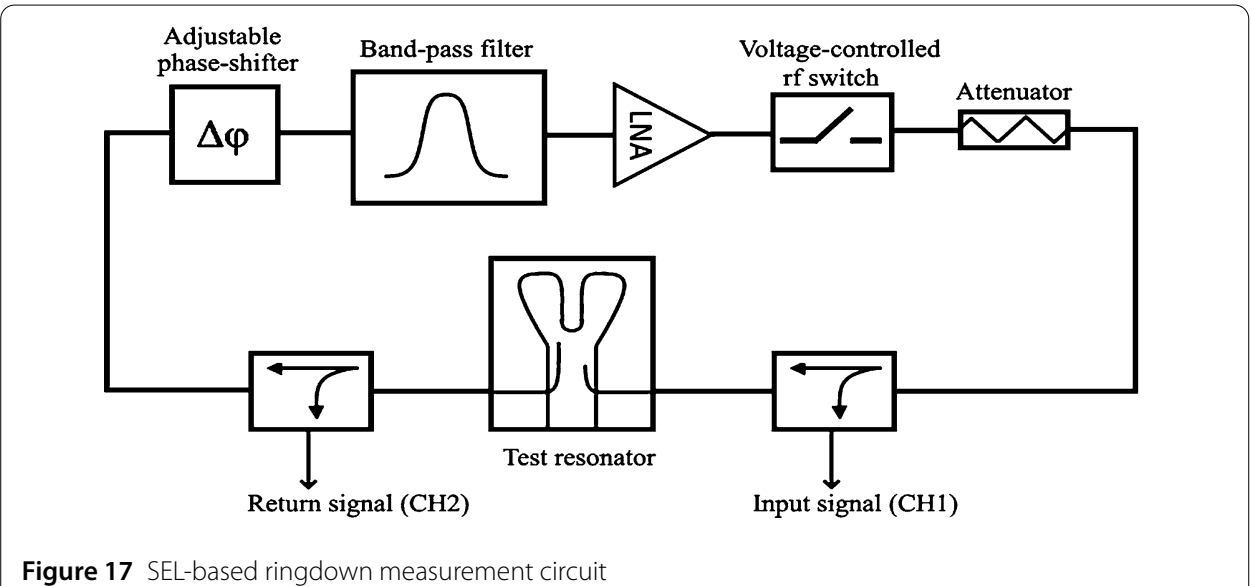

Figure 17 SEL-based ringdown measurement circuit

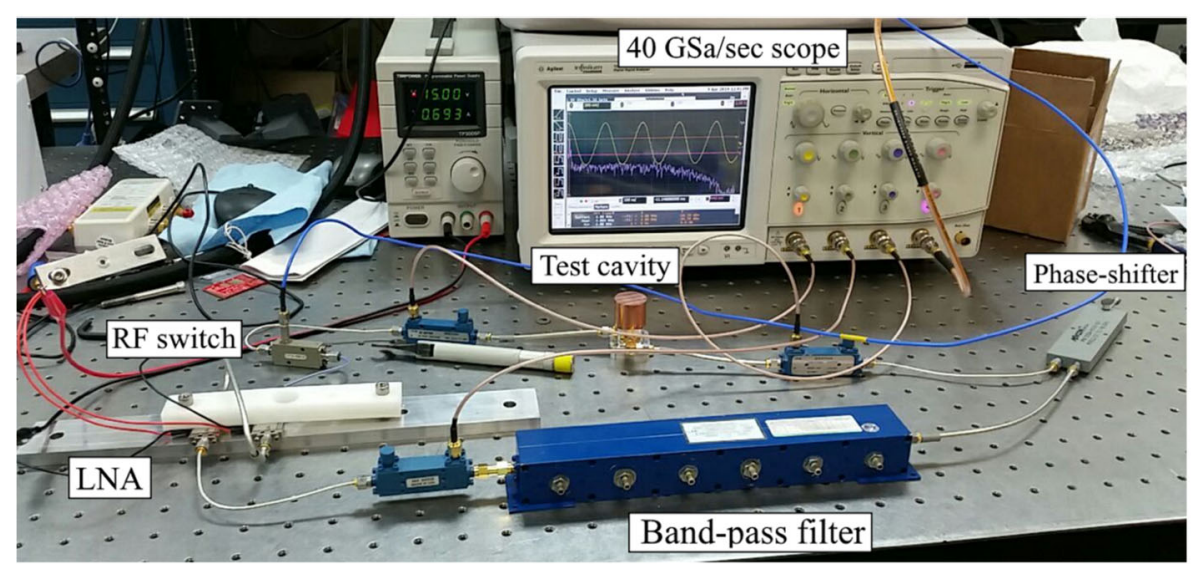

Figure 18 Picture of the room temperature test stand

Moving to the cryogenic tests, two etched resonators, one with the simple and the other with the optimized shape (shapes $b$ and $d$, as shown in Fig. 8) were used for Q-factor measurements in the quantum regime (10 $\mathrm{mK}$ temperature and a single-photon power level). The assembly is shown in Fig. 20.

We connected the resonators using the amplification chain installed inside the dilution refrigerator. The room temperature measurements of the installed resonators revealed that the resonant frequencies of both resonators increased by $200-250 \mathrm{MHz}$ after polishing. The frequency of the non-optimized resonator became $6226 \mathrm{MHz}$, while the frequency of the optimized resonator increased to $6098 \mathrm{MHz}$ (see Table 2, columns 2 and 4 for the frequencies before etching).

Both S-parameters and ringdown measurements were used to measure the loaded Qfactor $\left(Q_{L}\right)$, which is a combination of the resonator $\mathrm{Q}$-factor $\left(Q_{0}\right)$, external Q-factor of the coupling elements $\left(Q_{\mathrm{EXT}}\right)$ and the $\mathrm{Q}$-factor of the parasitic elements such as aluminum piece, seam losses, etc. ( $\left.Q_{\text {PAR }}\right)$ :

$$
\frac{1}{Q_{L}}=\frac{1}{Q_{0}}+\frac{1}{Q_{\text {EXT1 }}}+\frac{1}{Q_{\text {EXT2 }}}+\frac{1}{Q_{\text {PAR }}}
$$




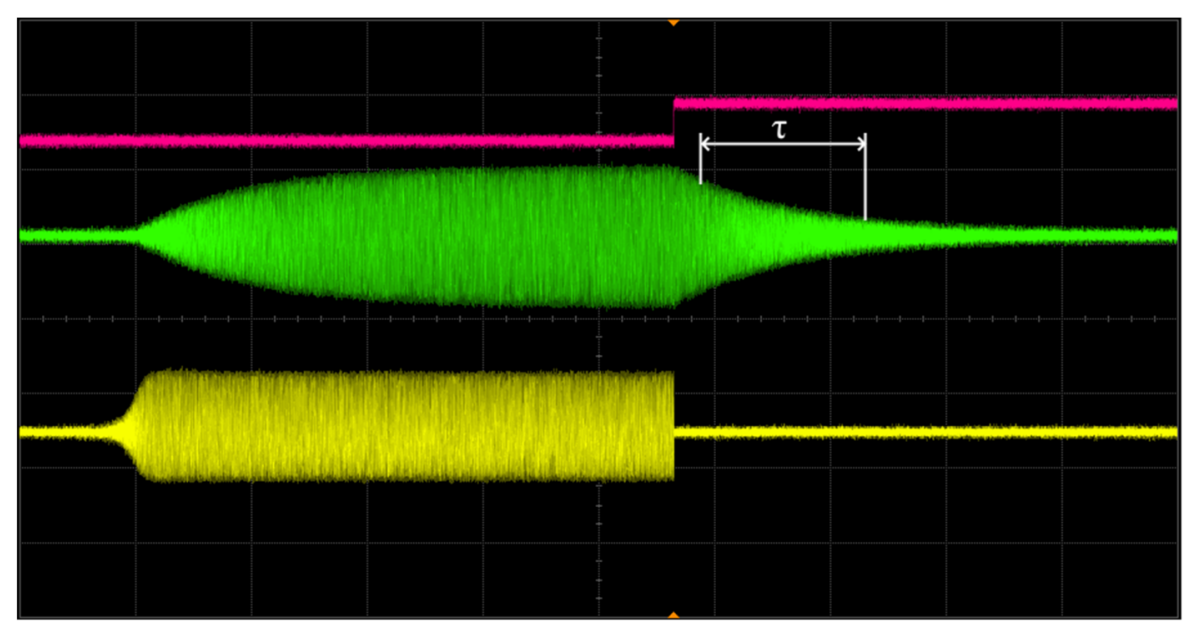

Figure 19 Ringdown waveforms captured using SEL: input rf pulse—yellow, return signal—green, rf switch control voltage - pink

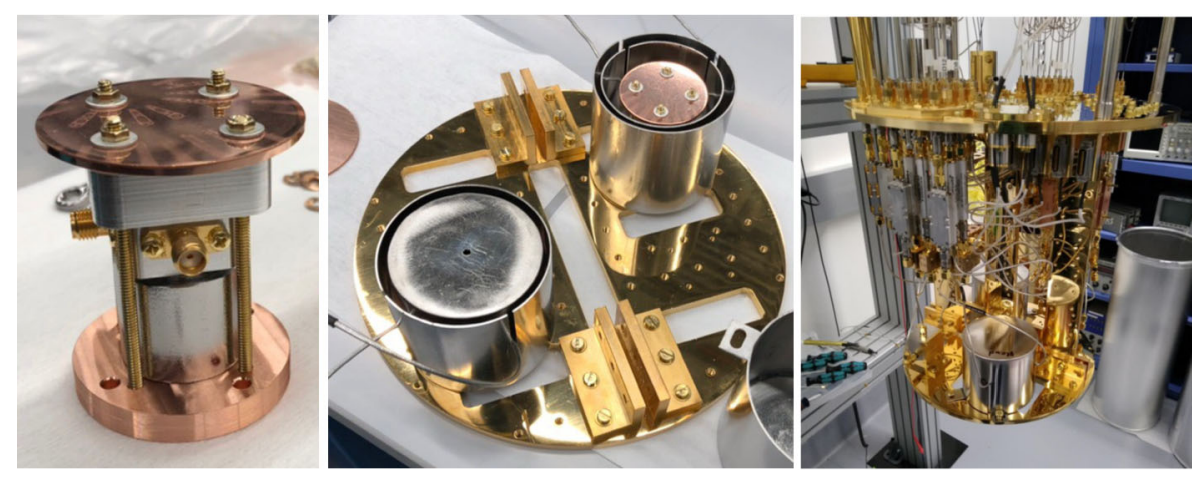

Figure $203 \mathrm{D}$ QWR resonator assembly (left), including Cryoperm shielding (center) attached to the $10 \mathrm{mK}$ stage of the dilution refrigerator at the University of Chicago (right)

Therefore, in order to measure the real Q-factor, we need to operate in the undercoupled regime: $Q_{\text {EXT }} \gg Q_{0}$. However, since $Q_{0}$ is not zero and $Q_{\text {EXT }}$ scales directly with the coupling pin length, we decided to perform a series of Q-factor measurements with different $Q_{\mathrm{EXT}}$, starting from $\sim 10^{5}$. One of the couplers was used to excite the signal in the resonator, and the other one was used as the field probe.

For the first measurement, SMA couplers lengths were adjusted to $Q_{\mathrm{EXT} 1}=4 \cdot 10^{5}$ and $Q_{\text {EXT2 }}=10^{7}$ according to the calibration curve shown in Fig. 16 . Then, on each successive run we increased the $Q_{\text {EXT }}$ by about an order of magnitude by reducing the coupler length. When the $Q_{0}$ starts to dominate in the $Q_{L}$, we should see no further changes in the measured Q-factor. Due to the project constraints, we had to limit the number of runs to three.

\section{Results and discussion}

For each measurement, the resonators were then cooled down to $10 \mathrm{mK}$ within 24 hours. It is worth mentioning that the resonators remained in the $100-150 \mathrm{~K}$ region for about 9 hours, long enough to be affected by Q-disease and have their Q-factor reduced [41, 42]. 

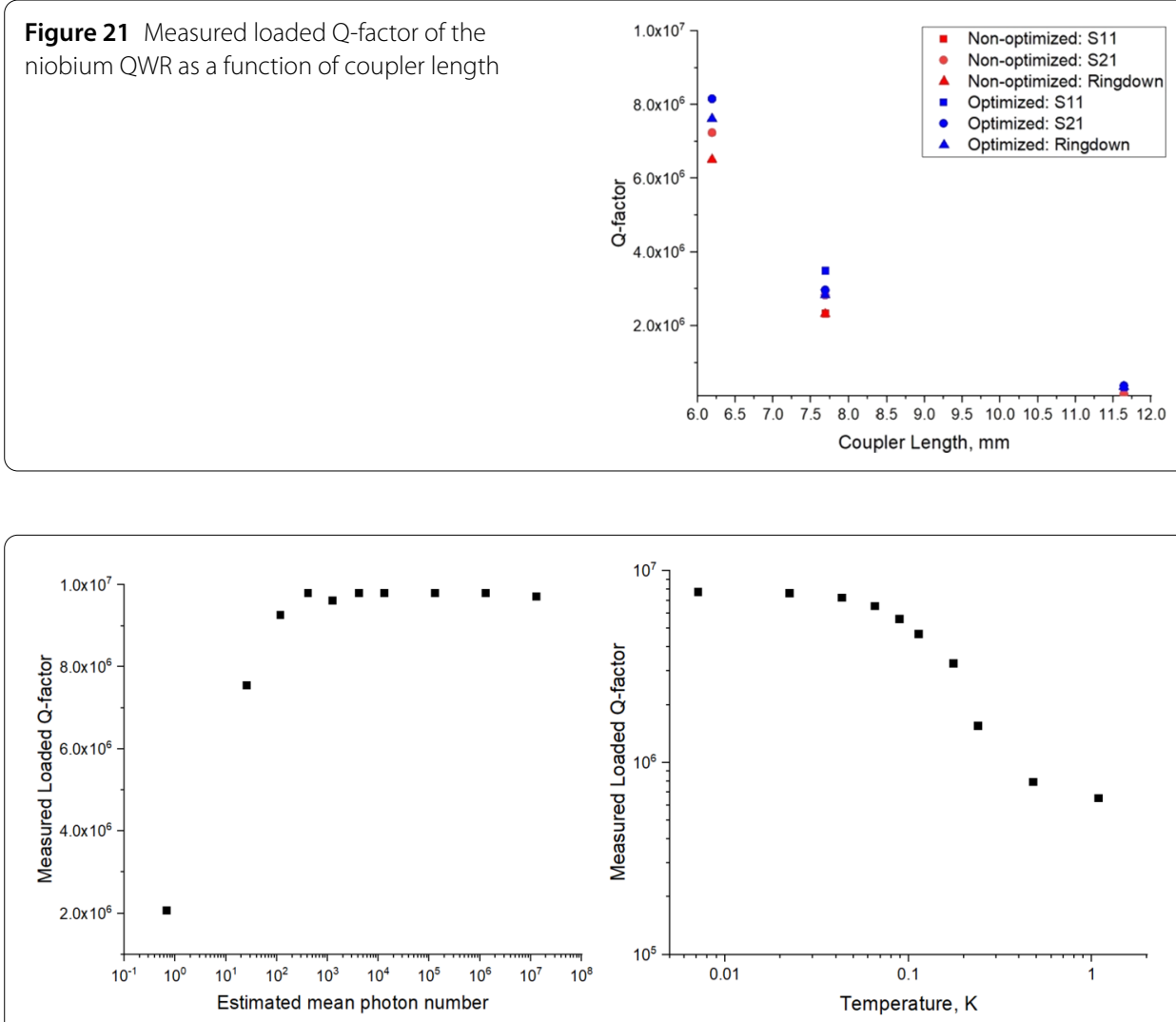

Figure 22 Q-factor of the niobium QWR as a function of power (left) and temperature (right)

The results for all three runs are summarized in Fig. 21. We can see a clear trend, where the Q-factor of the resonator with the optimized shape is higher by $\sim 25 \%$ than the Qfactor of the non-optimized resonators, measured by different techniques. Also, it is important to note that the Q-factor has not reached a plateau and grows exponentially with the length of the coupler, which may indicate that we are still in the overcoupled regime.

Finally, we have measured the Q-factor of the optimized resonator at single photon power levels and below and observed no change for the level of one photon or above. The Q-factor drops rapidly for levels below one photon. However, the signal to noise ratio is very low at these power levels. The results of the Q-factor measurements as a function of average input power level and temperature are shown in Fig. 22. The saturation of losses at higher power levels demonstrates that the low-power Q-factor is limited by losses in the dielectric layer and material imperfections [33, 43, 44].

\section{Conclusions}

RadiaBeam, in collaboration with the University of Chicago and Argonne National Laboratory, has developed a 3D SRF quarter-wave resonator with shape optimized for operation in the quantum regime. We used the known merits of SRF resonator design performance to demonstrate superior Q-factor performance of the optimized resonator. We have fabricated and etched two niobium resonators: one with simple and one with optimized shape. These prototype resonators were machined out of high-RRR niobium, chemically polished and high-pressure rinsed at Argonne National Laboratory and tested at the 
Figure 23 Comparison of Q-factors of the cavities with simple (non-optimized) and optimized geometries, measured by different methods and coupler lengths

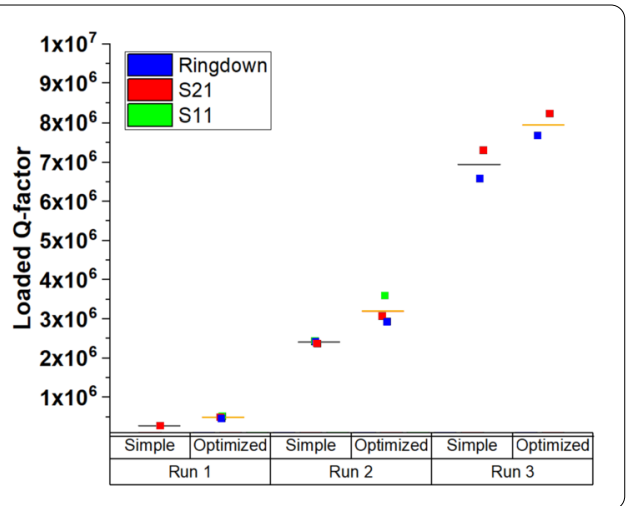

University of Chicago at $10 \mathrm{mK}$ up to 1 photon power levels. We used several methods to measure Q-factor, which demonstrated that the Q-factors of the resonators with optimized shaped are $\sim 25 \%$ higher than for the non-optimized resonators (see Fig. 23). This result demonstrates the proof-of-concept of the higher Q-factor due to shape optimization. Our future work will focus on improving the fabrication and surface processing of these resonators while incorporating new methods to limit the role surface oxides and nitrides play in reducing the cavity quality factors.

\section{Acknowledgements}

The authors would like to thank Dr. Alexander Romanenko from Fermi National Accelerator Laboratory for the important discussions about SRF qubit cavities. We would also like to acknowledge our RadiaBeam colleagues Peter Phillip and Daniel Villaseñor for their work on cavity fabrication, as well as Salime Boucher for constructive criticism of the manuscript.

Funding

This work was supported by the U.S. Department of Energy, Office of High Energy Physics, under SBIR grant DESC0018753.

\section{Abbreviations}

AC, alternating current; ANL, Argonne National Laboratory; BCP, buffered chemical polishing; BCS,

Bardeen-Cooper-Schrieffer theory; EM, electromagnetic; IR, infrared; LNA, low-noise amplifier; QC, quantum computers; QWR, quarter-wave resonant cavity; RF, radio frequency; RRR, residual-resistivity ratio; RT, room temperature; SBIR, Small Business Innovation Research; SEL, self-excited loop; SMA, SubMiniature connector version A; SRF, superconducting radiofrequency; VNA, vector network analyzer.

\section{Availability of data and materials}

The datasets used and/or analyzed during the current study are available from the corresponding author on reasonable request.

\section{Competing interests}

The authors declare that they have no competing interests.

\section{Authors' contributions}

The resonator design and optimization were done by KT and SK. Engineering design and fabrication studies were performed by RA, AM and PC. AS developed the SEL measurement stand. ZC performed the chemical treatment of the niobium cavities and consulted the authors in SRF technology. AC provided consultation in quantum technologies, qubit integration and hosted the experiment. The experiment was performed by KT, ED, AS and SK. The data was analyzed and interpreted by KT, ED, ZC, SK and AS. The manuscript was written and edited by SK, KT, ZC, RA, AS, ED and AC. The project was led by SK. All authors commented on the manuscript. All authors have read and approved the final manuscript.

\section{Author details}

${ }^{1}$ RadiaBeam Technologies LLC, Santa Monica, USA. ${ }^{2}$ National Research Nuclear University "MEPhl", Moscow, Russia.

${ }^{3}$ Pritzker School of Molecular Engineering, University of Chicago, Chicago, USA. ${ }^{4}$ Argonne National Laboratory, Lemont, USA. ${ }^{5}$ Present address: Brookhaven National Laboratory, Upton, USA.

\section{Publisher's Note}

Springer Nature remains neutral with regard to jurisdictional claims in published maps and institutional affiliations. 


\section{References}

1. Schumacher B. Phys Rev A. 1995:51:2738

2. Gershenfeld N, Chuang IL. Scientific American, June 1998.

3. Arute F et al. Nature. 2019;574(7779):505-10.

4. Zeh HD. Found Phys. 1970;1:69-76.

5. Amy M, et al. 2016. arXiv:1603.09383.

6. Makhlin Y, Schön G, Shnirman A. Nature. 1999;398(6725):305-7.

7. Ofek $N$ et al. Nature. 2016;536(7617):441-5.

8. Paik H et al. Phys Rev Lett. 2011;107(24):240501.

9. Bronn NT et al. Quantum Sci Technol. 2018;3(2):024007.

10. Béjanin JH et al. Phys Rev Appl. 2016;6(4):044010.

11. Josephson BD. Phys Lett. 1962;1(7):251-3.

12. Leek PJ et al. Phys Rev Lett. 2010;104:100504. 0911.4951

13. Sillanpaa MA, Park Jl, Simmonds RW. Nature. 2007;449:438-42.

14. Gambetta J et al. Phys Rev A. 2006;74:042318.

15. Houck AA et al. Phys Rev Lett. 2008;101:080502.

16. Reagor M et al. Phys Rev B. 2016;94:014506.

17. Posen S, Liepe M. Phys Rev Spec Top, Accel Beams. 2014;17:112001.

18. Kuhr S et al. Appl Phys Lett. 2007;90:164101.

19. Kutsaev S, et al. Quantum computing structures and resonators thereof. US Patent Application 62/837655. Filed April 23.

20. Padamsee H, Knobloch J, Hays T. RF superconductivity for accelerators. New York: Wiley; 1998.

21. Bardeen J, Cooper LN, Schrieffer JR. Phys Rev. 1957;108:1175.

22. Mattis DC, Bardeen J. Phys Rev. 1958;111:412.

23. Reagor M et al. Phys Rev B. 2016;94:014506.

24. Schultheiss TJ, et al. Proceedings of 2011 particle accelerator conference. New York, NY, USA.

25. Padamsee H. RF superconductivity: science, technology and applications (v. 2). Wiley-VCH; 2009. p. 1626.

26. https://www.3ds.com/products-services/simulia/products/cst-studio-suite/.

27. Romanenko A et al. Phys Rev Appl. 2020;13:034032.

28. Romanenko A. Employing SRF to boost coherence of 3D quantum systems. Talk at SRF'2019 conference, Dresden, Germany, 2019, https://srf2019.vrws.de/talks/thfub5_talk.pdf.

29. Daccà A et al. Appl Surf Sci. 1998;126:219-30.

30. Manuspiya H. Thesis, The Pennsylvania State University, 2003, p. 137.

31. Fuschillo N, Lalevic B, Annamalai NK. Thin Solid Films. 1975;30:145-54.

32. Knobloch J. Dissertation, Cornell University, August 1997, p. 26.

33. Martinis JM et al. Phys Rev Lett. 2005;95:210503

34. https://www.ansys.com/products/platform/multiphysics-simulation.

35. https://www.cryopermshielding.com/cryoperm-shielding.php.

36. Weast R. Handbook of chemistry and physics. 64th ed. Boca Raton: CRC Press; 1983. p. E-108.

37. https://alconox.com/.

38. Powers T. Theory and practice of cavity RF test systems. Proc. SRF'05. Ithaca: Cornell University; 2005.

39. Romanenko A, Schuster DI. Phys Rev Lett. 2017:119:264801.

40. Delayen J. Dissertation (Ph.D.), California Institute of Technology, 1979.

41. Gonnella D, Liepe M. Cool down and flux trapping studies on SRF cavities. In: Proc. of LINAC2014. Geneva, Switzerland. 2014.

42. Romanenko A et al. Appl Phys Lett. 2014;105:234103.

43. Megrant A et al. Appl Phys Lett. 2012;100:113510.

44. Wenner J. Appl Phys Lett. 2011;99:113513.

\section{Submit your manuscript to a SpringerOpen ${ }^{\circ}$ journal and benefit from:}

- Convenient online submission

- Rigorous peer review

- Open access: articles freely available online

- High visibility within the field

- Retaining the copyright to your article

Submit your next manuscript at $\gg$ springeropen.com 